\title{
Weierstrass condition for the general basic variational problem
}

\author{
Farhad Hïsseinov* \\ Bilkent University, Department of Economics, 06533, Ankara, Turkey
}

(MS received 17 February 1993. Revised MS received 10 February 1994)

The Weierstrass necessary condition for a multidimensional basic variational problem due to Hadamard is strengthened.

It is well-known that for the problems of variational calculus and optimal control the 'hidden convexity' property is inherent (see e.g. $[6,12]$ ). Usually, one points to N. N. Bogolubov's Theorem [2] on relaxation of the basic variational problem as a first result revealing this property. In fact, this property already had become apparent in the Weierstrass necessary condition in the classical variational calculus. Though these two classical results treat different type features of variational problems, they are closely related. Indeed, the Weierstrass condition obtained here is derived from the generalisation of Bogolubov's Theorem to the multidimensional case. In the geometric form, the Weierstrass condition states that, if $x_{0}(\cdot)$ is a strong local minimum for the basic variational problem with integrand $f\left(t, x, x^{\prime}\right)$, then the derivative $x_{0}^{\prime}(t)$ belongs to the convex frame of the function $f(t, x(t), \cdot): \mathbb{R} \rightarrow \mathbb{R}$ for all $t$ at which this derivative exists. Note that the same result is preserved in the case when the integrand $f$ depends on the scalar function $x(\cdot)$ of several variables. In the most general case, when the integrand $f$ depends on several functions $x_{1}(\cdot), \ldots, x_{m}(\cdot)$ of several variables $t^{1}, \ldots, t^{n}$, i.e. when $t=\left(t^{1}, \ldots, t^{n}\right) \in \Omega \subset \mathbb{R}^{n}$, the Weierstrass condition in its classical form ceases to be true, i.e. it is not necessary for the strong local minimum $x_{0}(\cdot)$ that the matrix $x_{0}^{\prime}(t)$ belongs to the convex frame of the function $f\left(t, x_{0}(t), \cdot\right): M^{n, m} \rightarrow \mathbb{R}$, where $M^{n, m}$ is the space of real matrices of dimension $m \times n$ with Euclidean structure. The Weierstrass condition for the most general case was discovered by Hadamard [9, 10], although the first strict proof was given by Graves [7]. In the geometrical form, that condition consists of the following: for any $t \in \Omega$, the matrix $x_{0}^{\prime}(t)$ belongs to the rank-one-convex (or $\Lambda$-convex) frame of the function $f\left(t, x(t, \cdot): M^{n, m} \rightarrow \mathbb{R}\right.$. Later different proofs were offered, e.g. [1, Theorem 3.4]. For relevant literature, see Ball [1].

Here we prove the Weierstrass condition, which strengthens the Hadamard-Graves condition. This condition consists of the following: if $x_{0}(\cdot)$ is a strong local minimum, then the matrix $x_{0}^{\prime}(t)$ belongs to the quasiconvex frame of the function $f\left(t, x_{0}(t), \cdot\right): M^{n, m} \rightarrow \mathbb{R}$ for almost every $t \in \Omega$. The Weierstrass condition was proved in [1, Theorem 3.1] under assumptions that included among others the smoothness

*On leave from Baku State University, Baku, 370602, Azerbaijan. 
at a point $x_{0}^{\prime}(t)$ of the integrand of the variational problem. In contrast to [1] as well as [7], we do not make any smoothness assumptions here. When a solution to the problem is known to be regular in a certain part of a domain $\Omega$, then, at the points in this part, the necessary condition found in this paper reduces to the quasiconvexity condition in [1] (see Theorem $1.8^{\prime}$ below). For conditions that guarantee regularity of a solution on an open subset of full measure, see Evans [5].

The methods of proof employed here, being based on results on the relaxation of variational problems, are principally different from those in the works cited above. The present methods point out the connections between two fields of variational calculus, relaxation theory and necessary conditions theory. This often yields more simple proofs, in particular, in the case of Corollary 1.11 which contains [1, Theorem 3.4].

Quasiconvex functions were discovered by C. B. Morrey [13] in connection with the investigation of lower semicontinuity of multiple integrals of the variational calculus. He proved that quasiconvexity of an integrand by a third variable $x^{\prime}$ is necessary and sufficient for the lower semicontinuity (with respect to a certain topology) of an integral functional. Note that if $m$ or $n=1$, then quasiconvexity is simply convexity and then this result of Morrey turns into the well-known classical result for this case. Furthermore, Morrey investigated quasiconvex functions. In particular, he proved that any quasiconvex function is rank-one convex, and raised the question of whether rank-one convexity implies quasiconvexity. This question has recently been solved negatively by Sverak [15]. It follows from this result that, in general, a quasiconvex frame is narrower than a rank-one convex frame. Therefore, the Weierstrass condition obtained here is stronger than Graves' result. Note also that in the light of Morrey's result on lower semicontinuity and recent results on lower semicontinuous relaxation of the general basic variational problem (see e.g. $[3,8]$ ), this condition becomes a natural and exact analogue of the classical Weierstrass condition.

Let $\bar{R}$ denote the set of real numbers extended by infinities $\pm \infty,|A|$ the Lebesgue measure of a measurable subset of $A$ of $R^{n}$. Let $G$ be an arbitrary bounded domain in $R^{n}$. Denote by $W_{\infty}^{1}\left(G, R^{m}\right)$ and $C^{\infty}\left(G, R^{m}\right)$, respectively, the spaces of Lipschitz functions and infinitely differentiable functions from $G$ to $R^{m}$, and denote by $W_{\infty, 0}^{1}\left(G, R^{m}\right)$ and $C_{0}^{\infty}\left(G, R^{m}\right)$, respectively, their subspaces consisting of functions vanishing on the boundary of $G$. Finally, denote by $\xi^{\prime}(t)$ the Jacobian matrix of a mapping $\xi: G \rightarrow R^{m}$ at the point $t \in G$.

DEFINITION 1.1. A function $f: M^{n, m} \rightarrow \bar{R}$ is called a quasiconvex function if

$$
f(p) \leqq|G|^{-1} \int_{G} f\left(p+\xi^{\prime}(t)\right) d t,
$$

for any fixed point $p \in M^{n, m}$ and an arbitrary vector-function $\xi(\cdot) \in W_{\infty, 0}^{1}\left(G, R^{m}\right)$ such that the function $f\left(p+\xi^{\prime}(\cdot)\right)$ is measurable and the integral on the right-hand side of the inequality (1.1) has a sense (i.e. assumes a certain value from $\overline{\mathbb{R}}$ ). The set of all such vector-functions will be denoted by $W_{\infty, 0}^{1}\left(G, \mathbb{R}^{m}, f, p\right)$.

REMARK 1.2. Usually, in the definition of a quasiconvex function, one supposes that (see $[3,13]$ ) the integral on the right-hand side of (1.1) exists for an arbitrary 
function $\xi(\cdot) \in W_{\infty, 0}^{1}\left(G, \mathbb{R}^{m}\right)$. On the contrary, we do not suppose it. Note that for any piecewise affine vector-function $\xi(\cdot)$, the function $f\left(p+\xi^{\prime}(\cdot)\right)$ is a step-function and consequently it is integrable.

DEFinition 1.3. Let $f: M^{n, m} \rightarrow \overline{\mathbb{R}}$ be an arbitrary function. A function $\tilde{f}: M^{n, m} \rightarrow \overline{\mathbb{R}}$ is called a quasiconvexification of the function $f$, if it is quasiconvex and not less than any quasiconvex function that does not exceed $f$. The quasiconvex frame of the function $f$ is called the set of points at which $\tilde{f}(p)=f(p)$. This set will be denoted by $Q F_{f}$.

The following theorem shows that, for an arbitrary function locally bounded from above, there exists its quasiconvexification, which is continuous. In particular, this result obviously implies continuity of any quasiconvex function locally bounded from above, which generalises the well-known property of convex functions. Note that a proof of the theorem on quasiconvexification under additional conditions was attempted by B. Dacorogna [3, Theorem 5]. But the proof of this theorem offered in [3] contains essential gaps. For example, the proof of the continuity of the function $Q_{k f}$ (see (1.2) below) is incorrect. The same proof is used in [4, p. 201, Theorem 1].

THEOREM 1.4. Let $f: M^{n, m} \rightarrow \overline{\mathbb{R}}$ be an arbitrary function locally bounded from above and let $K \subset \overline{\mathbb{R}}^{n}$ be a cube with sides parallel to the coordinate axes. For any point $p \in M^{n, m}, p u t$

$$
Q_{K} f(p)=\inf \left\{|K|^{-1} \int_{K} f\left(p+\xi^{\prime}(t)\right) d t: \xi(\cdot) \in W_{\infty, 0}^{1}\left(K, \mathbb{R}^{m}, f, p\right)\right\} .
$$

Then the function $Q_{K} f$ does not depend on the cube $K$, is continuous and is the quasiconvexification of $f$.

For the proof of Theorem 1.4, see [11].

Let $\Omega$ be an arbitrary bounded domain in $\mathbb{R}^{n}$. Let

$$
\bar{W}_{\infty}^{1}\left(\Omega, \mathbb{R}^{m}\right)=W_{\infty}^{1}\left(\Omega, \mathbb{R}^{m}\right) \cap C\left(\bar{\Omega}, \mathbb{R}^{m}\right)
$$

and $\Gamma$ be the boundary of $\Omega$. Let $f: \Omega \times \mathbb{R}^{m} \times M^{n, m} \rightarrow \mathbb{R}$ and $\varphi: \Gamma \rightarrow \mathbb{R}$ be arbitrary continuous functions. Consider the general basic variational problem

$$
\left\{\begin{array}{l}
J_{f}(x(\cdot))=\int_{\Omega} f\left(t, x(t), x^{\prime}(t)\right) d t \rightarrow \text { inf } \\
\left.x(\cdot)\right|_{\Gamma}=\varphi
\end{array}\right.
$$

Thus the set of admissible vector-functions in problem $(\mathrm{P})$ is $D=$ $\left\{x(\cdot) \in \bar{W}_{\infty}^{1}\left(\Omega, \mathbb{R}^{m}\right):\left.x(\cdot)\right|_{\Gamma}=\varphi\right\}$. Along with the problem $(\mathrm{P})$, consider another problem of the same kind:

$$
\left\{\begin{array}{l}
J_{\tilde{f}}(x(\cdot))=\int_{\Omega} \tilde{f}\left(t, x(t), x^{\prime}(t)\right) d t \rightarrow \mathrm{inf} \\
\left.x(\cdot)\right|_{\Gamma}=\varphi
\end{array}\right.
$$

where $\tilde{f}$ is the quasiconvexification of the function $f$ by the last argument. 
We now give a definition of relaxation of a variational problem in a suitable form.

DefinITION 1.5. Let $X$ be a topological space and $f_{1}, f_{2}$ be two functions defined on $X$. Then the variational problem inf $\left\{f_{2}(x): x \in X\right\}$ is said to be a relaxation of the variational problem inf $\left\{f_{1}(x): x \in X\right\}$, if for all $x \in X, f_{2}(x) \leqq f_{1}(x)$ and for any fixed $x \in X$, there exists a net $x_{\alpha} \in X(\alpha \in A)$ converging to $x$ such that $\lim _{\alpha} f_{1}\left(x_{\alpha}\right)=f_{2}(x)$.

THEOREM 1.6. In problem (P), let the set of admissible functions $D$ be endowed with the metric of uniform convergence. Then problem (PR) is a relaxation of problem (P).

The Proof of Theorem 1.6 easily follows from the main result of [11] (wherein 'extension' should read 'relaxation' throughout).

Let $g: M^{n, m} \rightarrow \bar{R}$ be an arbitrary function and let $\tilde{g}$ be its quasiconvexification. The set of points $p \in M^{n, m}$, at which $\tilde{g}(p)=g(p)$, is called the quasiconvex frame (or skeleton) (in the case $m=1$ or $n=1$ convex frame) of the function $g$. Denote this set by $Q F_{g}$.

Proposition 1.7. A point $p \in M^{n, m}$ belongs to the quasiconvex frame $Q F_{g}$ of a function $g: M^{n, m} \rightarrow \bar{R}$ if and only if for an arbitrary function $\xi(\cdot) \in C_{0}^{\infty}\left(K, R^{m}\right)$ the inequality

$$
g(p) \leqq|K|^{-1} \int_{\Omega} g\left(p+\xi^{\prime}(\tau)\right) d \tau
$$

is satisfied.

Proposition 1.7 follows almost immediately from Theorem 1.4.

THEOREM 1.8. (Weierstrass condition in geometrical form). Let a function $f: \bar{\Omega} \times \mathbb{R}^{m} \times M^{n, m} \rightarrow \mathbb{R}$ be continuous and assume that its quasiconvexification does not assume the value $-\infty$. Then if $x_{0}(\cdot)$ is a strong local minimum for problem $(\mathrm{P})$, the point $x_{0}^{\prime}(t)$ belongs to the quasiconvex frame $Q F_{f\left(t, x_{0}(t) .\right)}$ of the function $f\left(t, x_{0}(t), \cdot\right): M^{n, m} \rightarrow \mathbb{R}$, i.e.

$$
\tilde{f}\left(t, x_{0}(t), x_{0}^{\prime}(t)\right)=f\left(t, x_{0}(t), x_{0}^{\prime}(t)\right) \text { for almost all } t \in \Omega .
$$

Proof. It follows from Theorem 1.6 that $x_{0}(\cdot)$ is a strong local minimum for problem (PR) and

$$
J_{\tilde{f}}\left(x_{0}(\cdot)\right)=J_{f}\left(x_{0}(\cdot)\right)
$$

Suppose that relation (1.3) is not satisfied for almost all $t \in \Omega$. Then, for some subset $\Omega_{0} \subset \Omega$ of positive measure,

$$
\tilde{f}\left(t, x_{0}(t), x_{0}^{\prime}(t)\right)<f\left(t, x_{0}(t), x_{0}^{\prime}(t)\right), \quad \forall t \in \Omega_{0} .
$$

Since $\tilde{f}\left(t, x_{0}(t), x_{0}^{\prime}(t)\right) \leqq f\left(t, x_{0}(t), x_{0}^{\prime}(t)\right)$ for almost all $t \in \Omega$, it follows from the inequality (1.5) that $J_{\tilde{f}}\left(x_{0}(\cdot)\right)<J_{f}\left(x_{0}(\cdot)\right)$, which contradicts (1.4)

Theorem 1.8 can be reformulated as:

THEOREM 1.8'. Let the integrand $f$ be continuous and assume that its quasiconvexification does not assume the value $-\infty$. Then, if $x_{0}(\cdot)$ is a strong local minimum of 
problem $(\mathrm{P})$,

$$
f\left(t, x_{0}(t), x_{0}^{\prime}(t)\right) \leqq \int_{K} f\left(t, x_{0}(t), x_{0}^{\prime}(t)+\xi^{\prime}(\tau)\right) d \tau,
$$

for an arbitrary function $\xi(\cdot) \in C_{0}^{\infty}\left(K, \mathbb{R}^{m}\right)$ and for almost all $t \in \Omega$.

Definition 1.9. A function $g: M^{n, m} \rightarrow \mathbb{R}$ is rank-one convex if, for any two matrices $p_{1}, p_{2} \in M^{n, m}$ and any two numbers $\alpha_{1}, \alpha_{2}$ such that $\operatorname{rank}\left(p_{1}-p_{2}\right) \leqq 1$ and $\alpha_{1}, \alpha_{2}>0$, $\alpha_{1}+\alpha_{2}=1$,

$$
g\left(\alpha_{1} p_{1}+\alpha_{2} p_{2}\right) \leqq \alpha_{1} g\left(p_{1}\right)+\alpha_{2} g\left(p_{2}\right) .
$$

For an arbitrary function $g: M^{n, m} \rightarrow \mathbb{R}$, its rank-one convexification is called the greatest rank-one convex function $\hat{g}: M^{n, m} \rightarrow \mathbb{R}$ not exceeding $g$. The rank-one convex frame of a function $g$ is called the set of points $p \in M^{n, m}$ at which $\hat{g}(p)=g(p)$. This set will be denoted by $\Lambda F_{g}$.

THEOREM 1.10 (Morrey [13]). An arbitrary continuous quasiconvex function is rankone convex.

It follows immediately from this theorem that for an arbitrary function $g: M^{n, m} \rightarrow \overline{\mathbb{R}}$, $\hat{g} \geqq \tilde{g}$. Therefore $\Lambda F_{g} \subset Q F_{g}$. This inclusion, together with Theorem 1.8, yields the following corollary:

COROLLARY 1.11. If the assumptions of Theorem 1.8 are satisfied, then the point $x_{0}^{\prime}(t)$ belongs to the rank-one convex frame of the function $f\left(t, x_{0}(t), \cdot\right): M^{n, m} \rightarrow \mathbb{R}$, i.e.

$$
\hat{f}\left(t, x_{0}(t), x_{0}^{\prime}(t)\right)=f\left(t, x_{0}(t), x_{0}^{\prime}(t)\right) \quad \text { for almost all } t \in \Omega \text {. }
$$

Now we shall derive the result which is well known in the multidimensional variational calculus, namely the generalisation of the Weierstrass necessary condition due to Graves [7] from Corollary 1.11. In this connection, we shall need the following elementary result:

Proposition 1.12. Let $h, h_{1}: \mathbb{R} \rightarrow \mathbb{R}$ be two functions, with $h_{1}$ convex, $h_{1} \leqq h$ and $h_{1}(0)=h(0)$. Then if $h$ is differentiable at $0, h_{1}$ is also differentiable at 0 and their derivatives at 0 coincide, i.e. $h_{1}^{\prime}(0)=h^{\prime}(0)$.

COROLlary 1.13 (Graves [7]). If the assumptions of Theorem 1.8 are satisfied at any point $\left(t, x_{0}(t), x_{0}^{\prime}(t)\right)$ such that the function $f\left(t, x_{0}(t), \cdot\right)$ is differentiable at the point $x_{0}^{\prime}(t)$, then the Weierstrass E-function assumes non-negative values,

$$
\begin{aligned}
E\left(t, x_{0}(t), x_{0}^{\prime}(t), p\right)= & f\left(t, x_{0}(t), p\right)-f\left(t, x_{0}(t), x_{0}^{\prime}(t)\right)-\sum_{i, \alpha}\left(p_{\alpha}^{i}-\left(x_{0}^{\prime}(t)\right)_{\alpha}^{i}\right) \\
& \times f_{p_{\alpha}^{i}}\left(t, x_{0}(t), x_{0}^{\prime}(t)\right) \geqq 0, \quad \forall p \in M^{n, m}: \operatorname{rank}\left(p-x_{0}^{\prime}(t)\right) \leqq 1 .
\end{aligned}
$$

Proof. Let the function $f\left(t, x_{0}(t), \cdot\right)$ be differentiable at the point $p_{0}=x_{0}^{\prime}(t)$. Fix an arbitrary point $p \in M^{n, m}$ such that rank $\left(p-p_{0}\right) \leqq 1$. Consider the function $h(\lambda)=$ $f\left(t, x_{0}(t), p_{0}+\lambda\left(p-p_{0}\right)\right)$ of the scalar variable $\lambda$. Then the function $h_{1}(\lambda)=$ $\hat{f}\left(t, x_{0}(t), p_{0}+\lambda\left(p-p_{0}\right)\right)$ is convex, does not exceed the function $h(\cdot)$ and, by Corollary 1.11,

$$
h_{1}(0)=h(0)
$$


By Proposition 1.12 the function $h_{1}(\cdot)$ is differentiable at the point 0 and

$$
h_{1}^{\prime}(0)=h^{\prime}(0) \text {. }
$$

It is easily seen that

$$
h^{\prime}(0)=\sum_{i, \alpha} f_{p_{\alpha}^{i}}\left(t, x_{0}(t), p_{0}\right)\left(p_{\alpha}^{i}-p_{0 \alpha}^{i}\right) .
$$

We get from the relations (1.6)-(1.8) and inequality $h_{1}(1)-h_{1}(0) \geqq h_{1}^{\prime}(0)$, which follows from the convexity of $h_{1}$, that

$$
E\left(t, x_{0}(t), p_{0}, p\right)=f\left(t, x_{0}(t), p\right)-f\left(t, x_{0}(t), p_{0}\right)-\sum_{i, \alpha} f_{p_{\alpha}^{i}}\left(t, x_{0}(t), p_{0}\right)\left(p_{\alpha}^{i}-p_{0 \alpha}^{i}\right) \geqq 0 .
$$

The following simple reasoning shows how to construct an example of a variational problem, for which some admissible function satisfies the Graves condition but does not satisfy the Weierstrass condition obtained here. Let $f: M^{n, m} \rightarrow R$ be any rankone-convex but not quasiconvex function. Then there exists a point $p_{0} \in M^{n, m}$ such that $Q f\left(p_{0}\right)<f\left(p_{0}\right)$. A function defined as $x(t)=p_{0} t$ for $t \in \bar{\Omega}$ is an admissible function in the variational problem

$$
\int_{\Omega} f\left(x^{\prime}(t)\right) d t \rightarrow \inf ; \quad x(t)=p_{0} t \quad \text { for } t \in \Gamma
$$

with the required properties.

\section{Acknowledgment}

The author wishes to thank an anonymous referee for his valuable suggestions.

\section{References}

1 J. M. Ball. Existence theorems in nonlinear elasticity. Arch. Rational Mech. Anal 63 (1977), $337-403$.

2 N. N. Bogolubov. Sur quelques methods novelles dans le calculus des variations. Ann. Math. Pura Appl. (4) 7 (1930), 249-71.

3 B. Dacorogna. Quasiconvexity and relaxation for nonconvex problems in the calculus of variations. J. Funct. Anal. 46 (1982), 102-18.

4 B. Dacorogna. Direct Methods in the Calculus of Variations (Berlin: Springer, 1989).

5 L. C. Evans. Quasiconvexity and partial regularity in the calculus of variations. Arch. Rational Mech. Anal. 95 (1986), 227-52.

6 R. V. Gamkrelidze. Foundations of Optimal Control (Tbilisi: Tbilisi University Press, 1975).

7 L. M. Graves. The Weierstrass condition for multiple integral variation problems. Duke Math. J. 5 (1939), 656-60.

8 F. V. Hüsseinov (F. V. Guseinov). Lower semicontinuous extension of the fundamental problem of calculus of variations. Mat. Zametki 45 (3) (1989), 43-52; English translation: Math. Notes 45 (3) (1989), 210-17.

9 J. Hadamard. Sur une question de calcul des variations. Bull. Soc. Math. France 30 (1902), $253-6$.

10 J. Hadamard. Leçons sur la Propagation des Ondes (Paris: Hermann, 1903).

11 F. V. Hüsseinov. Continuity of quasiconvex functions and theorem on quasiconvexification. Izv. Akad. Nauk Azerbaidzhan. SSR Ser. Fiz.-Tekhn. Mat. Nauk 8 (1988), 17-23.

12 A. D. Ioffe and V. M. Tikhomirov. Theory of Extremal Problems (Moscow: Nauka, 1974).

$13 \mathrm{Ch}$. B. Morrey. Quasiconvexity and the lower semi-continuity of multiple integrals. Pacific J. Math. 2 (1952), 25-53.

14 Ch. B. Morrey. Multiple Integrals in the Calculus of Variations (Berlin: Springer, 1966).

15 V. Sverak. Rank-one convexity does not imply quasiconvexity. Proc. Roy. Soc. Edinburgh Sect. A (1992), 185-9. 